\title{
Towards a general model for predicting minimal metal concentrations co-selecting for antibiotic resistance plasmids
}

\author{
Sankalp Arya ${ }^{1}$, Alexander Williams ${ }^{1}$, Saul Vazquez Reina ${ }^{2}$, Charles W. Knapp ${ }^{3}$ \\ Jan-Ulrich Kreft ${ }^{4}$, Jon L. Hobman ${ }^{5}$, Dov J. Stekel ${ }^{1 *}$, \\ 1 Division of Agricultural and Environmental Sciences, School of Biosciences, University \\ of Nottingham, Sutton Bonington Campus, Loughborough, LE12 5RD, UK \\ 2 Gateway Building, Sutton Bonington Campus, University of Nottingham, Sutton \\ Bonington LE12 5RD, UK \\ 3 Civil \& Environmental Engineering, University of Strathclyde, James Weir Bldg., \\ 5.03K, 75 Montrose Street, Glasgow, G1 1XJ, UK \\ 4 School of Biosciences \& Institute of Microbiology and Infection \& Centre for \\ Computational Biology, University of Birmingham, Edgbaston, Birmingham, B15 2TT, \\ UK \\ 5 Division of Microbiology, Brewing and Biotechnology, School of Biosciences, \\ University of Nottingham, Sutton Bonington Campus, Loughborough, LE12 5RD, UK
}

Towards a general model for predicting minimal metal concentrations co-selecting for antibiotic resistance plasmids

Sankalp Arya ${ }^{1}$, Alexander Williams ${ }^{1}$, Saul Vazquez Reina ${ }^{2}$, Charles W. Knapp ${ }^{3}$ Jan-Ulrich Kreft ${ }^{4}$, Jon L. Hobman ${ }^{5}$, Dov J. Stekel ${ }^{1 *}$,

1 Division of Agricultural and Environmental Sciences, School of Biosciences, University of Nottingham, Sutton Bonington Campus, Loughborough, LE12 5RD, UK

2 Gateway Building, Sutton Bonington Campus, University of Nottingham, Sutton Bonington LE12 5RD, UK

3 Civil \& Environmental Engineering, University of Strathclyde, James Weir Bldg., 5.03K, 75 Montrose Street, Glasgow, G1 1XJ, UK

4 School of Biosciences \& Institute of Microbiology and Infection \& Centre for Computational Biology, University of Birmingham, Edgbaston, Birmingham, B15 2TT, UK

5 Division of Microbiology, Brewing and Biotechnology, School of Biosciences, University of Nottingham, Sutton Bonington Campus, Loughborough, LE12 5RD, UK

Towards a general model for predicting minimal metal concentrations co-selecting for antibiotic resistance plasmids

Sankalp Arya ${ }^{1}$, Alexander Williams ${ }^{1}$, Saul Vazquez Reina ${ }^{2}$, Charles W. Knapp ${ }^{3}$ Jan-Ulrich Kreft ${ }^{4}$, Jon L. Hobman ${ }^{5}$, Dov J. Stekel ${ }^{1}$,

1 Division of Agricultural and Environmental Sciences, School of Biosciences, University of Nottingham, Sutton Bonington Campus, Loughborough, LE12 5RD, UK 2 Gateway Building, Sutton Bonington Campus, University of Nottingham, Sutton Bonington LE12 5RD, UK 
3 Civil \& Environmental Engineering, University of Strathclyde, James Weir Bldg., 5.03K, 75 Montrose Street, Glasgow, G1 1XJ, UK

4 School of Biosciences \& Institute of Microbiology and Infection \& Centre for

Computational Biology, University of Birmingham, Edgbaston, Birmingham, B15 2TT, UK

5 Division of Microbiology, Brewing and Biotechnology, School of Biosciences, University of Nottingham, Sutton Bonington Campus, Loughborough, LE12 5RD, UK

* dov.stekel@nottingham.ac.uk

\begin{abstract}
Many antibiotic resistance genes co-occur with resistance genes for transition metals, such as copper, zinc, or mercury. In some environments, a positive correlation between high metal concentration and high abundance of antibiotic resistance genes has been observed, suggesting co-selection due to metal presence. Of particular concern is the use of copper and zinc in animal husbandry, leading to potential co-selection for antibiotic resistance in animal gut microbiomes, slurry, manure, or amended soils. For antibiotics, predicted no effect concentrations have been derived from laboratory measured MICs and some minimal selective concentrations have been investigated in environmental settings. However, minimal co-selection concentrations for metals are difficult to identify. Here, we use mathematical modelling to provide a general mechanistic framework to predict minimal co-selective concentrations for metals, given knowledge of their toxicity at different concentrations. We apply the method to copper $(\mathrm{Cu})$, Zinc $(\mathrm{Zn})$, mercury $(\mathrm{Hg})$, lead $(\mathrm{Pb})$ and silver $(\mathrm{Ag})$, predicting their minimum co-selective concentrations in mg/L (Cu: 5.5, Zn: 1.6, Hg: 0.0156, Pb: 21.5, Ag: 0.152). To exemplify use of these thresholds, we consider metal concentrations from slurry and slurry-amended soil from a UK dairy farm that uses copper and zinc as additives for feed and antimicrobial footbath: the slurry is predicted to be co-selective, but not the slurry-amended soil. This modelling framework could be used as the basis for defining standards to mitigate risks of antimicrobial resistance applicable to a wide range of environments, including manure, slurry and other waste streams.
\end{abstract}

\title{
Author summary
}

\section{Introduction}

The persistence and spread of antimicrobial resistance (AMR) is a major global threat, with at least 700,000 deaths per year attributed to bacterial infections by drug-resistant strains world-wide [1]. Reduction of antibiotic use, or cessation of use of some veterinary antibiotics, is seen as critically important to mitigate the threat of AMR. A classic example of this strategy has been the banning of avoparcin in poultry production, e.g. Germany and Denmark in 1995, other EU countries by 1997 and Taiwan in 2000. The success of this ban can be exemplified with Norwegian poultry farms showing high abundance (99\%) of vancomycin-resistant enterococci (VRE) in farms exposed to avoparcin prior to the ban and lower abundance $(11 \%)$ in samples from unexposed farms [3], while in Taiwan there was a decrease from $25 \%$ farms having vancomycin-resistant enterococci (VRE) in 2000 to $8.8 \%$ farms in 2003 [2].

However, the continued presence of resistant strains suggests that there may be other factors that promote persistence of antibiotic resistance genes (ARGs). One of these factors is co-selection: selective pressure exerted by a toxicant that maintains 
other coupled ARGs. This coupling can occur in different ways: (i) co-resistance, i.e., multiple genes encoding resistance against different antibiotics and metals which are on the same genetic element; (ii) cross-resistance, i.e., the same mechanism (e.g., efflux pumps) providing resistance against multiple toxicants; (iii) co-regulation, which is the coordinated response to the presence of either antibiotic or metal, this activates mechanisms necessary for the resistance against the other or both [4]. High metal concentration provides co-selective pressure for certain antibiotics. For example, Lee et al. (2005) showed that the $m d t$ operon, which encodes for a multidrug resistance efflux pump in E. coli was up-regulated in response to excess zinc [5]. Resistance to antibiotics is also enriched in response to metal shock loading 6, 7], or due to long-term exposure to metal 8, 9]. However, most data only provides indirect evidence co-selection due to metal presence, by providing evidence of co-occurrence of metal and antibiotic resistance, in many environments, including oral and intestinal [10], sludge bioreactors [11], marine [12] and soil sediments [13].

Selective pressure can be caused at concentrations lower than the minimal inhibitory concentration (MIC). The FAO and WHO support the concept of minimum selective concentration (MSC) for antibiotics, i.e., a threshold concentration above which the resistance genes are selected. MSCs are available for antibiotics, based on standard MICs, through both empirical and modelling approaches [14 16, 18], although environmental studies show the issue to be much more complex [17]. However, co-selection pressure due to transition metals might mean that in some environments, ARGs could be selected for and maintained in a bacterial community even with antibiotic concentrations below MSC. Metal and antibiotic resistance genes co-occur in environments where the metal contamination 19,20 is sufficiently high to provide co-selection pressure for persistence and proliferation of antibiotic resistance 10 21 23]. The notion of Minimal Co-Selective Concentrations (MCSCs) for transition metals was introduced by Seiler and Berendonk [13, who identified possible thresholds based upon observations of metal concentrations in a range of environments. The lack of appropriate MCSCs has been highlighted by the FAO and WHO 24]; indeed a rigorous and consistent approach to defining MCSCs could be used, alongside toxicity, to inform suitable standards for metal concentrations in agriculture or environmental contexts.

We address this research gap using a mathematical modelling approach. Models can help to understand and predict the impact of co-selection under different scenarios, and have already helped in understanding factors associated with AMR emergence and spread such as mutation rates [25], antibiotic consumption [26], water troughs on farms 27] as well as quantifying the importance of factors such as conjugation [28]. Models have also accurately predicted MIC values of $\beta$-lactams against MRSA [29]. One of the few mathematical models for co-selection studied the concentration of resistant bacteria in the Poudre River in Colorado and determined that external input and selection pressure solely due to tetracycline was insufficient to explain the observed levels of resistant bacteria. A co-selection model, on the other hand, which considered both tetracycline and metal concentrations reproduced the observed data [30].

In this study, we developed a general model to quantify the effect of metal concentrations on persistence of resistance in a bacterial population, using an approach that can be applied to any metal and any environment, given knowledge of its toxicity in that environment. We compare the results from deterministic (applicable to large well-mixed populations) and stochastic versions (applicable to small populations where random events may be significant) of the same model. We then analyse the effects of metal toxicity and plasmid fitness cost on the persistence of resistance in each version of the model. The model allows us to identify MCSCs for transition metals and how they depend on the toxicity of the metal and the fitness cost of carrying resistance. We show that both deterministic and stochastic versions of the model provide similar results, 
with resistance lost only at high fitness costs and sufficiently dilute metal concentrations, i.e. low toxicity. However, the stochastic model does suggest a higher chance of persistence for several months without antibiotic selection. Finally, we demonstrate the use of the MSCSs by applying them to measurements of copper and zinc concentrations in dairy slurry and slurry-amended soil on the same farm.

\section{Materials and methods}

\section{Model description}

The purpose of this analysis is to understand how the persistence of resistance genes is dependent on the fitness cost of carrying the resistance genes (for example carried on a plasmid) and the selective pressure from metals being present in the environment. We also investigate how deterministic and stochastic modelling paradigms impact upon the results. The models describe a generalised process of conjugation transferring the resistance genes, how bacterial growth is affected by the fitness cost of plasmid carriage (if present), and how death is affected by the concentration of metal in the environment.

We model a small (micro-scale) volume element representative of a larger system. The complete macro-scale ecosystem can be considered to be made up of replicates of the modelled domain 31,57 . The starting bacterial population is of primarily resistant bacteria (99.32\%), without antibiotic present, because we are interested in the persistence of resistance rather than the spread of resistance. The deterministic version of the model is described by ordinary differential equations (ODEs) Eq (1)-(2). As in Baker et al. 28, bacterial growth is defined by a logistic growth terms, affected by the fitness cost $(\alpha)$ of the plasmid for the resistant cells. Conjugation uses a classic Sensitive-Infected-Resistant/Recovered (SIR) model formulation for plasmid transfer, with "infection" of sensitive cells (S) by resistant cells (R) with rate constant $\beta$. The differences from the Baker et al. model [28] are the inclusion of death of sensitive $\left(\delta_{S}\right)$ and resistant bacteria $\left(\delta_{R}\right)$ - at different rates due to the resistance to metal - and plasmid loss $(\epsilon)$ due to segregation upon cell division.

$$
\begin{array}{r}
\frac{d S}{d t}=r\left(1-\frac{N}{N_{\max }}\right) S-\delta_{S} S-\frac{\beta S R}{N}+r\left(1-\frac{N}{N_{\max }}\right)(1-\alpha) \epsilon R \\
\frac{d R}{d t}=r\left(1-\frac{N}{N_{\max }}\right)(1-\alpha)(1-\epsilon) R-\delta_{R} R+\frac{\beta S R}{N}
\end{array}
$$

where $N=S+R$. The same model structure can be described by a set of discrete events which define the stochastic simulation algorithm (SSA). Table 1 provides the details of this SSA. Each event has a reaction rate which is the same as the rates defined in ODEs Eq (1)-(2).

We estimated the parameters for bacterial death rate for both resistant and sensitive bacteria under different metal concentrations using the metal toxicity values for $E$. coli provided by Ivask et al. 32 and Equation (3). We used the SCO (Social Cognitive Optimization) evolutionary solver in LibreOffice Calc to estimate the unknown parameters $E_{\max }$ (maximum death rate due to metal), MIC (Minimum Inhibitory Concentration) and $\mathrm{H}$ (Hill coefficient) for all metals.

$$
\delta_{S}=\delta_{R}+\frac{E_{\max } M^{H}}{M I C^{H}+M^{H}}
$$

where, $M$ is the metal concentration. Once the parameters were estimated, the concentrations of copper and zinc measured using the ICP-MS techniques, for both 
Table 1. Stochastic Simulation Algorithm for Eq (1) - (2)

\begin{tabular}{|l|l|l|}
\hline Event & Description & Rate \\
\hline$S \rightarrow 2 S$ & Growth of sensitive bacteria & $r\left(1-\frac{N}{N_{\max }}\right) S$ \\
\hline$S \rightarrow$ & Death of sensitive bacteria & $\delta_{S} S$ \\
\hline$R \rightarrow 2 R$ & Growth of resistant bacteria & $r\left(1-\frac{N}{N_{\max }}\right)(1-\alpha) R$ \\
\hline$R \rightarrow$ & Death of resistant bacteria & $\delta_{R} R$ \\
\hline$S \rightarrow R$ & Conjugation & $\frac{\beta S R}{N}$ \\
\hline$R \rightarrow S+R$ & Plasmid loss due to segregation & $r\left(1-\frac{N}{N_{\max }}\right)(1-\alpha) \epsilon R$ \\
\hline
\end{tabular}

The table lists the events describing the lifecycle of the bacteria as well as the processes of conjugation and plasmid loss. The rates for the different events are the same as in the ODE version of the model.

slurry and slurry treated soil, were used to calculate the ratio of death rate of sensitive to resistant bacteria $(\zeta)$.

\section{Numerical solutions of model}

For the deterministic model, differential equations were simulated using the $\mathrm{R}[33$ deSolve package 34 LSODA algorithm and sensitivity analysis was performed using the rootSolve [35 36], doParallel 37] and foreach [38] R packages. For the parameter sensitivity analysis using the stochastic model, we used COPASI [39], and created shell scripts to run each parameter combination one thousand times. The output of each run was then imported into $\mathrm{R}$ to produce parameter sensitivity graphs with the ggplot2 $[40]$ package, with 5-dimensional data expressed as two spatial dimensions and three colour dimensions, using an RGB combination for each point associated with each parameter combination, red for persistence of resistance, blue for loss of resistance, and green for total cell death. For example, if out of 1000 runs of the stochastic version of the model, 300 runs predicted persistence of resistance and 700 runs loss of resistance, then a colour $30 \%$ red and $70 \%$ blue would be plotted.

For example environments, we took the measured values of copper and zinc concentrations in slurry and slurry amended soil and calculated the death rate ratio of sensitive to resistant populations based on the metal toxicity values of $E$. coli [32], to check resistance fixation conditions in each environment, as described in the Model Overview section.

\section{Multi-elemental analysis by ICP-MS}

\section{Slurry}

Slurry samples $(2 \mathrm{~mL})$ were acid digested on a hot plate using $6 \mathrm{~mL}$ Primar Plus grade $\mathrm{HNO}_{3}(68 \%)$ and $2 \mathrm{~mL} \mathrm{H} \mathrm{O}_{2}$ (Thermo Fisher Scientific, Loughborough, UK). Samples were diluted with Milli-Q water $(18.2 \mathrm{M} \Omega \mathrm{cm})$ to $50 \mathrm{~mL}$ and syringe filtered to $<0.2 \mu \mathrm{m}$ (Merck-Millipore, Burlington, USA) prior to analysis by inductively coupled plasma mass spectrometry (icapQ model; Thermo Fisher Scientific, Bremen, Germany). Samples were introduced (flow rate $1.2 \mathrm{~mL} \mathrm{~min}^{-1}$ ) from an autosampler (Cetac ASX-520) incorporating an ASXpress rapid uptake module through a perfluoroalkoxy (PFA) Microflow PFA-ST nebuliser (Thermo Fisher Scientific, Bremen, Germany). Sample processing was undertaken using Qtegra software (Thermo-Fisher Scientific) utilizing external cross-calibration between pulse-counting and analogue detector modes when required. The ICP-MS was run employing two operational modes with in-sample switching between a collision cell (i) charged with He gas with kinetic energy 
discrimination (KED) to remove polyatomic interferences and (ii) using $\mathrm{H}_{2}$ gas as the cell gas. The latter was used only for Se determination. Peak dwell times were $100 \mathrm{~ms}$ with 150 scans per sample.

Internal standards, used to correct for instrumental drift, were introduced to the sample stream on a separate line (equal flow rate) via the ASXpress unit and included Sc $(10 \mu \mathrm{g} / \mathrm{L}), \operatorname{Ge}(10 \mu \mathrm{g} / \mathrm{L}), \operatorname{Rh}(5 \mu \mathrm{g} / \mathrm{L})$ and $\operatorname{Ir}(5 \mu \mathrm{g} / \mathrm{L})$. The matrix used for internal standards, calibration standards and sample dilution was $2 \%$ Primar Plus grade $\mathrm{HNO}_{3}$ with $4 \%$ methanol (to enhance ionization of some elements such as Se).

Calibration standards included (i) a multi-element solution with $\mathrm{Ag}, \mathrm{Al}, \mathrm{As}, \mathrm{Ba}, \mathrm{Be}$, Cd, Ca, Co, Cr, Cs, Cu, Fe, K, Li, Mg, Mn, Mo, Na, Ni, P, Pb, Rb, S, Se, Sr, Ti, Tl, U, $\mathrm{V}$ and $\mathrm{Zn}$, in the range 0 to $100 \mu \mathrm{g} / \mathrm{L}(0,20,40,100 \mu \mathrm{g} / \mathrm{L})$ (Claritas-PPT grade CLMS-2 from SPEX Certiprep Inc., Metuchen, NJ, USA); (ii) a bespoke external multi-element calibration solution (PlasmaCAL, SCP Science, France) with $\mathrm{Ca}, \mathrm{Mg}, \mathrm{Na}$ and $\mathrm{K}$ in the range $0-30 \mathrm{mg} / \mathrm{L}$ and (iii) a mixed phosphorus, boron and sulphur standard made in-house from salt solutions $\left(\mathrm{KH}_{2} \mathrm{PO}_{4}, \mathrm{~K}_{2} \mathrm{SO}_{4}\right.$ and $\left.\mathrm{H}_{3} \mathrm{BO}_{3}\right)$.

\section{Soil}

For extractable macro- and micro-elemental analysis, $1 \mathrm{~g}$ of soil was suspended in $9 \mathrm{~mL}$ of $1 \mathrm{M} \mathrm{NH}_{4} \mathrm{NO}_{3}$ and mixed thoroughly by agitation using a rotary shaker for 1 hour. Subsequently samples were centrifuged and $1 \mathrm{~mL}$ of the resulting supernatant was diluted in $9 \mathrm{~mL}$ of $2 \%$ nitric acid. Finally, samples were passed through a $0.22 \mu \mathrm{m}$ filter before being loaded for inductively coupled plasma mass spectrometry (ICP-MS; Thermo-Fisher Scientific iCAP-Q; Thermo Fisher Scientific, Bremen, Germany)

\section{Parameters used in the models}

The parameters were taken to match the model parameters of Baker et al. [28], where possible; other parameter values were taken from references in Table 2 The estimated death rates for metals from the metal toxicity model are also listed. For sensitivity analysis, the ratio of death rates $(\zeta)$ and the fitness cost of carrying the plasmid with resistance genes are varied over a range. We also increased and decreased transfer frequency and probability of segregational loss, to see the effects of these two parameters on the output.

\section{Results}

\section{To persist or not to persist and the role of chance}

In these simulations, we consider the persistence of the resistant strains following withdrawal of antibiotic, but in the continued presence of metal. Therefore the population starts at $99.3 \%$ resistant cells with only a small concentration of sensitive cells. We consider a microcosm of this population to understand the co-selective behaviour of metal presence as well as the stochasticity. We vary the fitness cost and ratio of death rates (sensitive/resistant) and measure the percentage of resistant population in the microcosm. Fig 1 shows the change in ratio of resistant cells over a period of 100 days, with each curve signifying a different set of values for the fitness cost and death rate ratio. Fig 1 (a) shows the deterministic model output and Fig 1 (b) shows the stochastic model output. As we can see, depending on the parameter values, deterministic model leads to lower number of resistance bacteria remaining after at least a period of 40 days. Fig 1(b), shows the output of stochastic model, with different colours representing different value set of the fitness cost and death rate ratio. We can see that stochasticity results in different outputs - either loss or persistence of resistance 
Table 2. All parameters used in equations (1)-(3).

\begin{tabular}{|c|c|c|c|}
\hline Parameter & Description & Value (Range) & Source \\
\hline$r$ & Specific growth rate & $0.5 \mathrm{~h}^{-1}$ & $41-43$ \\
\hline$N_{\max }$ & Carrying capacity of liquid slurry & $6.71 \times 10^{7} \mathrm{CFU} / \mathrm{L}$ & 44 \\
\hline$\delta_{R}$ & $\begin{array}{l}\text { Death rate of resistant bacteria (base } \\
\text { rate) }\end{array}$ & $0.025 \mathrm{~h}^{-1}$ & 45 \\
\hline$\delta_{S}$ & Death rate of sensitive bacteria & $\zeta \cdot \delta_{R}$ & varied \\
\hline$\zeta$ & $\begin{array}{l}\text { Ratio of } \delta_{S} \text { to } \delta_{R} \text { affected by metal } \\
\text { concentration }\end{array}$ & $1.0(1.0-10.0)$ & varied \\
\hline$\alpha$ & Fitness cost for carrying resistance & $\mathbf{0 . 1}(0-0.99)$ & $42,46,47$ \\
\hline$\beta$ & Conjugation rate & $0.001 h^{-1}$ & 47,48 \\
\hline$\epsilon$ & Plasmid loss probability & 0.000144 & $\overline{49}$ \\
\hline Volume & Volume of microcosm & $2.5 \times 10^{-6} \mathrm{~L}$ & Assumed \\
\hline \multicolumn{4}{|c|}{ Copper $\left(\mathrm{CuSO}_{4}\right)$} \\
\hline MIC & Minimum inhibitory concentration & $212.79 \mathrm{mg} / \mathrm{L}$ & Estimated \\
\hline$E_{\max }$ & Maximum death rate due to metal & $1.74 \mathrm{~h}^{-1}$ & Estimated \\
\hline $\mathrm{H}$ & Hill coefficient & 1.54 & Estimated \\
\hline \multicolumn{4}{|c|}{ Zinc $\left(\mathrm{ZnSO}_{4}\right)$} \\
\hline MIC & Minimum inhibitory concentration & $2760.31 \mathrm{mg} / \mathrm{L}$ & Estimated \\
\hline$E_{\max }$ & Maximum death rate due to metal & $1.37 \mathrm{~h}^{-1}$ & Estimated \\
\hline $\mathrm{H}$ & Hill coefficient & 0.72 & Estimated \\
\hline \multicolumn{4}{|c|}{ Mercury $\left(\mathrm{HgCl}_{2}\right)$} \\
\hline MIC & Minimum inhibitory concentration & $1.85 \mathrm{mg} / \mathrm{L}$ & Estimated \\
\hline$E_{\max }$ & Maximum death rate due to metal & $5.89 \mathrm{~h}^{-1}$ & Estimated \\
\hline $\mathrm{H}$ & Hill coefficient & 1.44 & Estimated \\
\hline \multicolumn{4}{|c|}{ Lead $\left(\mathrm{Pb}\left(\mathrm{NO}_{3}\right)_{2}\right)$} \\
\hline MIC & Minimum inhibitory concentration & $\mathbf{1 7 2 8 . 7} \mathrm{mg} / \mathrm{L}$ & Estimated \\
\hline$E_{\max }$ & Maximum death rate due to metal & $18.74 \mathrm{~h}^{-1}$ & Estimated \\
\hline $\mathrm{H}$ & Hill coefficient & 1.82 & Estimated \\
\hline \multicolumn{4}{|c|}{ Silver $\left(\mathrm{AgNO}_{3}\right)$} \\
\hline MIC & Minimum inhibitory concentration & $0.48 \mathrm{mg} / \mathrm{L}$ & Estimated \\
\hline$E_{\max }$ & Maximum death rate due to metal & $2.42 \mathrm{~h}^{-1}$ & Estimated \\
\hline $\mathrm{H}$ & Hill coefficient & 5.19 & Estimated \\
\hline
\end{tabular}

- with same parameter values (same colour lines), however, there is also a decrease in time for loss of resistance to less than 10 days in certain cases, when in deterministic model it requires atleast 40 days for $50 \%$ loss.

\section{Effect of toxicity and fitness cost on persistence of resistance}

In order to evaluate persistence of resistance due to co-selection, we carried out a sensitivity analysis for two parameters, metal toxicity and fitness cost for plasmid carriage. We measured the time for the resistant population to drop from $99.32 \%$ to $50 \%$ (Fig 2). The dominant outcome for higher levels of metal toxicity (i.e. higher concentrations) and lower levels of fitness cost is persistence of resistance (grey zone in Fig 22. The coloured zone indicates loss of resistance from blue (rapid loss) to red (slower loss). A key feature is the steep rise in the boundary between the two zones: as the level of toxicity increases, the minimum fitness cost required for loss of resistance also increases sharply. Although we have covered the full theoretical range of fitness cost for completeness, the typical realistic range is from 0.1 to 0.3 [42,46, 47], with 


\section{Ratio of resistant bacteria over time}

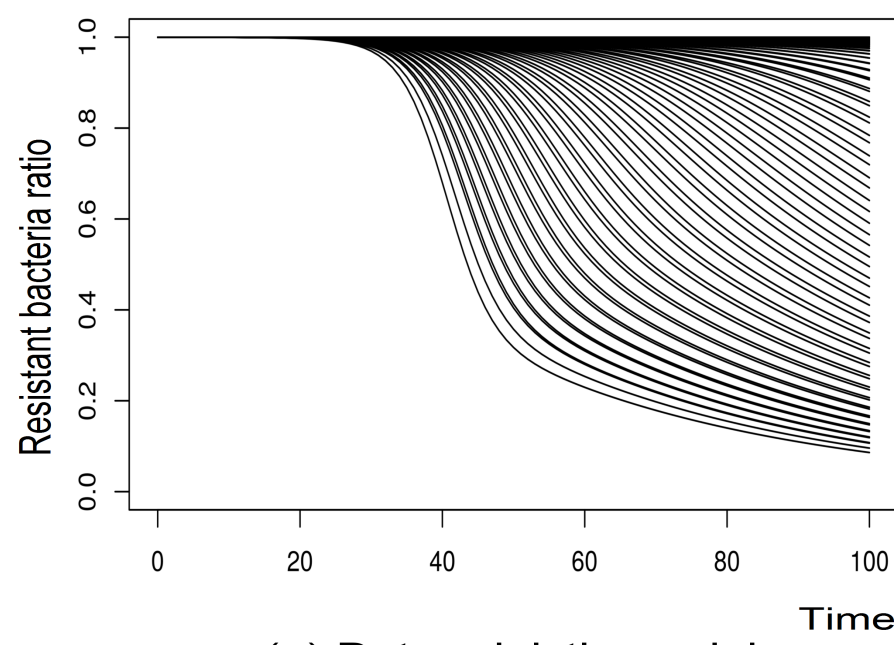

(a) Deterministic model

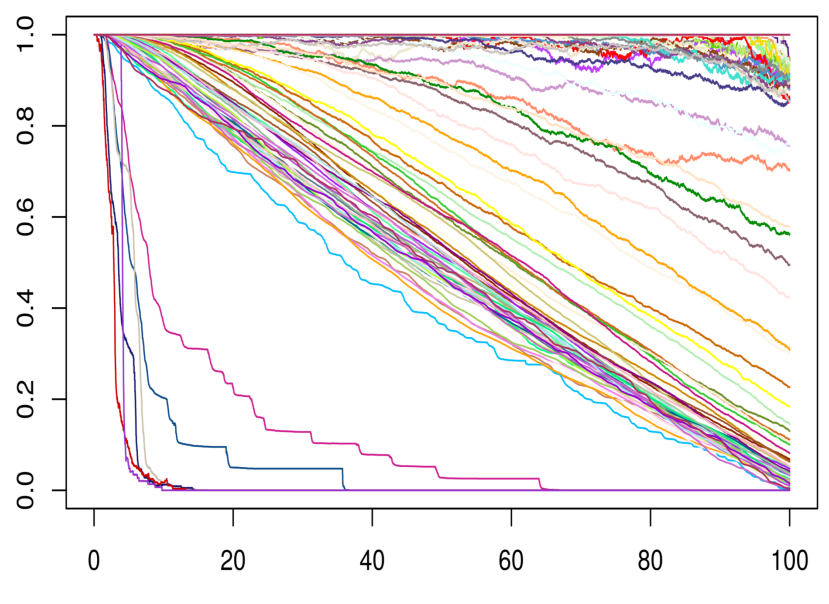

(b) Stochastic model

Fig 1. Amalgamation of time series curves. The figure shows time series curves up to 100 days for each of the variations of fitness cost and death rate ratio of sensitive to resistant bacteria, both for (a) deterministic and (b) stochastic model. The two modelling paradigms show different behaviours. In the deterministic model, resistance typically persists for approximately 40 days, and then may persist or not according to the parameter values. In the stochastic model, there is stochastic variation in both whether and for how long resistance persists even with the same parameter values (same colour lines). In either case, maintenance of resistance can be seen on this time scale.

variations occurring during the growth of bacteria.

Compared to the deterministic model with a single outcome for a set of parameter values, a stochastic model may result in different outcomes on repeated runs with same parameter values. Thus, in order to assess the impact of stochasticity, the probability of different outcomes was coded as different colours on the RGB scale, with red denoting resistant bacteria at more than $50 \%$, blue denoting resistant bacteria less than $50 \%$ and green denoting loss of all bacteria (Fig 3). Thus, a mix of these colours at a point signifies that the same parameter combination resulted in different outcomes. As can be seen in Fig 3(a), after 100 days there is a greater chance of persistence (red) rather than loss (blue), whereas after a 1000 days (i.e. 3 years) or $10^{5}$ days (chosen as a very long period to allow the model to equilibriate), there was a clear pattern of resistance loss or persistence, similar to the pattern for the deterministic model. Thus, the outcomes in Fig. 1 with loss of resistance in less than 100 days, have a low probability, as inferred from Fig 3(a). Even then, the fitness cost required for such loss, is higher than typical costs of stable plasmids $42,46,47$.

Our previous work highlighted the importance of gene transfer rate on spread of resistance 28. Therefore, to assess the robustness of the outcomes shown in Fig 2 to changes in the conjugation rate parameter, we conducted similar sensitivity analyses with conjugation frequency 10 times higher (Fig \) and lower (Fig [). The result with lower conjugation frequency is almost identical, probably because conjugation is not an important factor when resistance is already high. On the other hand, with increased conjugation frequency, there is increased persistence of resistance. However, the default 


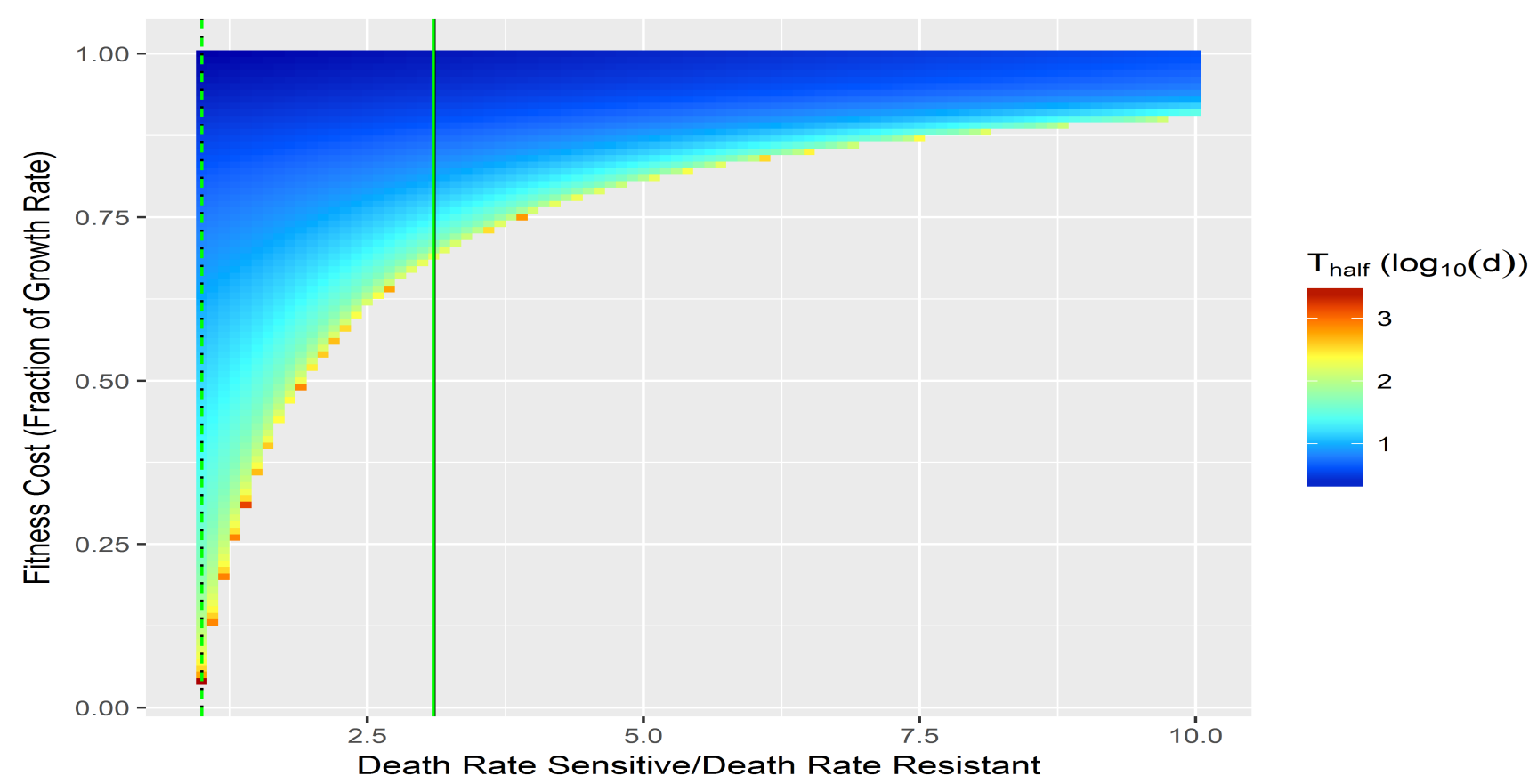

Fig 2. Sensitivity analysis of the deterministic model. The figure shows the number of days for the resistant population to drop to $50 \%$ total population from the same starting point of $99.32 \%$ resistant population, in the absence of antibiotic. The x-axis represents metal toxicity in terms of ratio of sensitive against resistant bacterial death rate. As can be seen, co-selection pressure causes persistence of resistance at low fitness cost and high metal toxicity. Loss of resistance is seen only with high fitness cost or with no metal - ratio of death rates equal to 1 . The vertical lines represent the death rate ratio for copper (green) and zinc (black) concentrations in the example environments of slurry (solid lines) and slurry amended soil (dashed lines at almost identical x- coordinates) as measured by the method mentioned before. For both metals, the observed concentrations lead to similar death rate ratios, with higher chance of persistence in slurry than slurry amended soil.

conjugation frequency $\left(0.001 \mathrm{~h}^{-1}\right)$ is already at the upper end of observed values, so higher values are unlikely to be realistic. Therefore, we are confident that the results shown are robust to variations in transfer rate. We also varied the value of probability of plasmid loss due to segregation, showing that a higher loss probability speeds up loss of resistance (Fig \), whereas a lower loss probability has similar outcome (Fig [). Again, higher rates of plasmid loss are not likely to be especially relevant because of plasmid addiction systems, and so we are confident that our results are robust to variations in this parameter too. Changing growth rate had no effect on the output (data not shown).

\section{Estimating minimum co-selective concentrations (MCSCs)}

We presented model outcomes in terms of the ratio of death rates at different metal concentrations (Fig 2); these can be used to predict minimal co-selection concentrations for specific metal, bacterial and plasmid combinations, using a metal toxicity model (Eq. $(3)$ ), and knowledge of the fitness cost of carriage. For these calculations, we used a fitness cost of 0.25 , which is within the reasonable range of expected values 42, 46 47, although this could be reduced to produce more stringent MCSCs. Thus, the death rate ratio selected was 1.25. Using the estimated parameter values, we calculated the MCSC value based on this ratio. The data is presented in Table 3 for copper, zinc, mercury, lead and silver using $E$. coli as an examplar. 


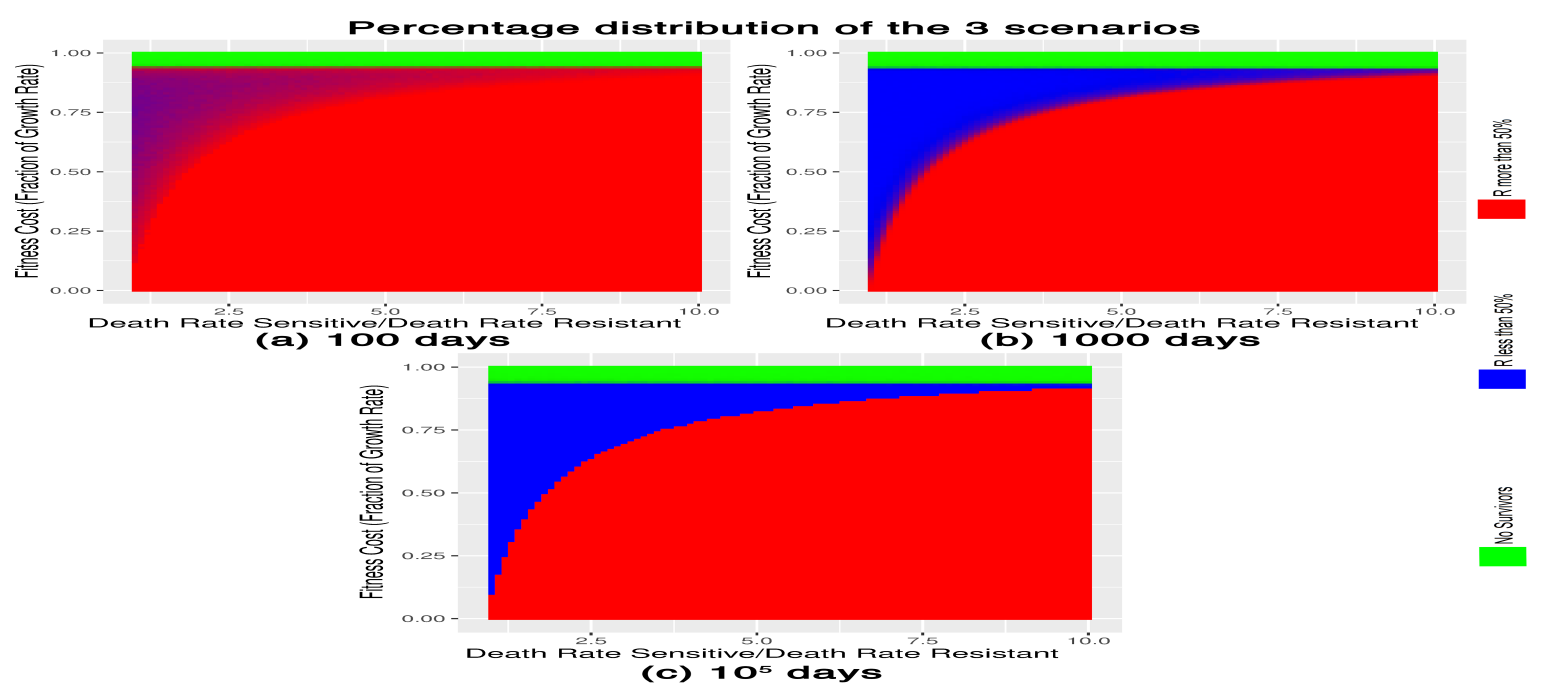

Fig 3. Sensitivity analysis of the stochastic model. The figure shows the percentage distribution of three outcomes occurring in the stochastic simulations. Each outcome is represented by a different colour. The three panels show different time points in the simulations, 100, 1000 and 100,000 days. In the short term, there is a greater probability of persistence of resistance as after 100 days, cases with relatively high fitness cost show very little loss of resistance but after 1000 days, we see a similar pattern as seen for the ODE simulations.

Table 3. Metal MCSC and measured concentrations

\begin{tabular}{|l|l|l|l|}
\hline Metal & MCSC (mg/L) & Farm Soil $(\mathbf{m g} / \mathbf{k g})$ & Dairy Slurry $(\mathbf{m g} / \mathbf{L})$ \\
\hline Copper $(\mathrm{Cu})$ & 5.5 & 0.068 & 22.32 \\
Zinc $(\mathrm{Zn})$ & 1.6 & 0.16 & 32.16 \\
Mercury $(\mathrm{Hg})$ & 0.0156 & NA & NA \\
Lead $(\mathrm{Pb})$ & 21.5 & NA & 0.047 \\
Silver $(\mathrm{Ag})$ & 0.152 & NA & 0.00026 \\
\hline
\end{tabular}

The MCSC threshold is calculated by assuming death rate ratio of 1.25, which corresponds to fitness cost of 0.25 for predicted loss of resistance in Fig. 2. A metal concentration above this threshold is predicted to provide co-selective pressure and lead to persistence of resistance. With our farm example data, concentrations of copper and zinc are predicted to be coselective in the slurry, but not slurry amended soil. Measured concentrations of lead and silver in the slurry are predicted not to be coselective.

In reference to our example environments, measured zinc concentrations are 32.158 $\mathrm{mg} / \mathrm{L}$ (slurry) and $0.3 \mathrm{mg} / \mathrm{L}$ (soil), and copper concentrations are $22.317 \mathrm{mg} / \mathrm{L}$ (slurry) and $0.17 \mathrm{mg} / \mathrm{L}$ (soil). Therefore the metal concentration in slurry is above the MCSC for both metals, hence this will be classified as a co-selective environment; however, the metal concentration is below the MCSC for both metals in slurry-amended soil, so that would not be a co-selective environment. Similarly, the measured concentrations of lead and silver in slurry are both below the MCSC threshold and so these metals are not predicted to be co-selective.

\section{Discussion}

Several studies have shown that there is a correlation between presence of metals and abundance of antibiotic resistance genes (ARGs) in soil, including in Scotland [50], Western Autralia [51] and urban soils from Belfast area [52]. These correlations are 
indicative of co-selection due to metal presence, but do not prove a causal link. The model described in this study provides a mechanistic insight into the different factors which drive co-selection. Although both deterministic and stochastic versions were defined with similar assumptions and parameter values, the inherent assumptions about the biological processes in each methodology are different and hence might lead to different results. The deterministic version shows that loss of resistance genes or resistant bacteria is only possible in low toxicity (lower death rate ratio) environment, or, in the rare case of cost prohibitive plasmids (high fitness cost). Most AMR phenomena are observed in large scale environments such as guts, tanks, fields, farms, hospitals 53, and the deterministic model can provide a reasonable approximation for prediction of the behaviour of large scale populations if they are, or can be considered to be, well-mixed. However, most environments are comprised of smaller, diverse, microscopic bacterial communities, and so deterministic models may be less realistic; thus stochasticity can play an important role 57. The stochastic model in this work leads to a similar general conclusion of the effects of metal toxicity and fitness cost, but stochasticity leads to a greater chance of persistence over a shorter (reasonable) time-scale of antibiotic absence.

Importantly, the models we developed can be used to predict minimal co-selective concentrations (MCSCs) for transition metals. These predictions can help inform metal concentration thresholds for environments in which antibiotic resistant bacteria are likely to be present - provided other organisms don't have toxicity values much lower than predicted MCSCs. Animal agriculture is a prime example due to both metal and antibiotic use. The MCSC determined here is low compared to the permissible concentrations set by the various guidelines. For example, the ALS Environmental guideline for soil concentrations has the maximum permissible value for copper and zinc set at $80 \mathrm{mg} / \mathrm{kg}$ and $200 \mathrm{mg} / \mathrm{kg}$, respectively, for $\mathrm{pH}$ between $5.0-5.4$ and $200 \mathrm{mg} / \mathrm{kg}$ and $450 \mathrm{mg} / \mathrm{kg}$ respectively at $\mathrm{pH}$ of 7.1 or higher [54]. The same report gives the concentrations for mercury and lead at $1 \mathrm{mg} / \mathrm{kg}$ and $300 \mathrm{mg} / \mathrm{kg}$, respectively, for $\mathrm{pH} 5.0$ or higher. Similarly, Seiler and Berendonk 13. suggest an MCSC for soil of $117 \mathrm{mg} / \mathrm{kg}$ fresh weight for copper. Our study would indicate that current guidelines provided for soil metal concentrations are not sufficiently stringent and might provide a co-selective environment.

On the other hand, guidelines for maximum permissible concentration (MPC) for water - either freshwater, saltwater or groundwater (but not dirty water) - suggest extremely low metal concentrations. Taking the example of the report by National Institute of Public Health and the Environment Bilthoven, The Netherlands, we see that MPCs provided for copper are 1.5, 1.4 and $2.4 \mu \mathrm{g} / \mathrm{L}$ [55], which is 1000 fold lower than the MCSC value estimated by the model. This might be due to more sensitive toxicity levels of other organisms found in these environments or the use of these sources for drinkable water. Similar values are reported for other metals as well. These figures are very similar to the MCMCs suggested by Seiler and Berendonk [13] for copper and zinc $(1.5 \mu \mathrm{g} / \mathrm{L}$ and $19.61 \mu \mathrm{g} / \mathrm{L}$ respectively), but these concentrations may be difficult to apply to dirty water (e.g. slurry).

The current model does not explicitly account for changes in bioavailability of metal, which may cause change in toxicity values. For example, $\mathrm{Cr}^{3+}$ ions are less toxic than chromate and which form they occur in is dependant on environmental conditions [56]. Thus, this speciation (physiochemical form of metal) can affect the toxicity of the metals. Also, determination of element bio-availability remains rather unpredictable and highly contingent on adsorption dynamics, absorption within soil particles, flocculation, ion exchange, precipitation and complexation reactions. While classical geochemical Pourbaix relationships can provide insight about possible interactions based on $\mathrm{pH}$ and Eh (redox), a lot remains dependent on surface character and affinities, especially soil 
organic matter, water, salinity and temperature. Elevations in $\mathrm{pH}$ tend to reduce

solubility, and the presence of carbon dioxide tends to promote carbonate precipitation; Eh reductions enhance sulfide precipitation; whereas salinity (or presence of multiple ions) tends to mobilize the metals.

The BCR483 extraction $\left(\mathrm{NH}_{4} \mathrm{NO}_{3}\right)$ provides a good representation of trace element mobility [?], and represent the bio-availability from the sludge amended soils. The acid/peroxide extractions represent oxidizable forms and probably over-estimate metal lability, but does reflect the fraction associated with organic carbon, which can be highly dynamic in terms of complexation and solubility.

Copper availability tends to be highly dependent on organic matter content, to the extent that $\mathrm{kd}$ values for $\mathrm{Cu}^{2+}$ tended to be independent of $\mathrm{pH}$ (when >5.5) and driven by organic carbon [?]. While sludge amendments can enhance organic matter content in soils, and $\mathrm{Cu}$ binding [?,?], the presence of dissolved organic matter can mobilize the copper [?]. Lead strongly binds to organic matter, especially humic at $\mathrm{pH}>4$. Shifts between anoxia and oxic conditions may induce periods of solubility, but remain reactive to sulfide precipitation. Solubility values tend to be associated with $\mathrm{pH}$ under low carbon and sulfur environmental matrices; in case here, it is likely to be associated with the nature of the organic matter. Zinc can become insolubilised with sulfide at reducing conditions and can form relatively insoluble carbonate precipitates at higher pH. However, lability of zinc best correlates with total zinc concentrations [?], rather than precipitation reactions. Dissociation reactions are similar whether applied as sludge or as a salt [?]. Silver in the environment, while extremely toxic, is relatively insoluble. It strongly binds to organic matter and oxides within the soils, to the point that desorption is considered negligible.

The toxicity model defined in this study, uses the bioavailable metal concentration values and the Hill equation to calculate the death rate. While this empirical approach fits the data, more mechanistic approaches could be appropriate. Moreover, the toxicity values that are reported by Ivask et al. [32] are not based on the environment and do not take into account the interaction between different metals or metal and other biocides.

Despite these assumptions, the model can be applied to a large number of environments, with relatively minor changes. However, in more complex environments, spatial heterogeneity and stochasticity may become more important [57. Another complexity that is missing and might provide further insight into the role of metals towards co-selection is the addition of multiple metals. Environmental contaminants occurring in a mixture is an observed and quantified norm. Thus, the presence of multiple metals might affect their bioavailability/toxicity. Data exists to show the correlation between contaminant mixture and ARGs [58], but, this only proves that there is co-occurrence of multiple toxicants and a higher abundance of ARGs. A mechanistic understanding of co-selection due to multiple toxicants is missing and might provide interesting results. Similar data, if available for other co-selective agents like biocides, QACS, etc. can be used to calculate the death rate and thus understand their potential for antibiotic resistance co-selection.

In conclusion, our model shows that co-selective pressure can maintain antibiotic resistance in microbial communities, even in the absence of antibiotic. It provides a general approach for setting appropriate standards for transition metal contamination, especially for environments where antibiotic resistance is likely to be important, e.g. in livestock farming, and monitoring those environments against those standards. It also implicates the importance of developing technologies for removing metals from such environments [59].

\section{Supporting information}


S1 Fig. Conjugation rate is $\mathbf{0 . 0 1}$, i.e., 10 times more than for Fig 2, We can see this leads to greater chances of persistence of resistance, even in situations with no selection pressure.

S2 Fig. Conjugation rate of 0.0001 (10 times lower). Shows very little difference compared to Fig 2

S3 Fig. 10 times higher probability of loss of resistance carrying plasmid due to segregation (0.00144). The affect is seen in the time required for loss of resistance. Most of the situation that lead to persistence are not affected, but where loss is likely the time required for loss of $50 \%$ of resistance from population is reduced by approximately a factor of 10 , as the red colour corresponds to $10^{3}$ days, when before it was $10^{4}$ days.

S4 Fig. 10 times lower probability of loss of plasmid due to segregation (0.0000144). Similar results to that seen in Fig 2

\section{Acknowledgments}

\section{References}

1. O'Neill J. Tackling Drug-resistant Infections Globally: Final Report and Recommendations; 2016. Available from: https://amr-review.org.

2. Lauderdale TL, Shiau YR, Wang HY, Lai JF, Huang IW, Chen PC, et al. Effect of banning vancomycin analogue avoparcin on vancomycin-resistant enterococci in chicken farms in Taiwan: Brief report. Environmental Microbiology.

2007;9(3):819-823. doi:10.1111/j.1462-2920.2006.01189.x.

3. Borgen K, Simonsen G, Sundsfjord A, Wasteson Y, Olsvik Ø, Kruse H. Continuing high prevalence of VanA-type vancomycin-resistant enterococci on Norwegian poultry farms three years after avoparcin was banned. Journal of Applied Microbiology. 2000;89(3):478-485.

4. Baker-Austin C, Wright MS, Stepanauskas R, McArthur JV. Co-selection of antibiotic and metal resistance; 2006. Available from: https:

//www.sciencedirect.com/science/article/pii/S0966842X06000515

5. L J L, J A B, R K P. Genome-wide transcriptional response of chemostat-cultured Escherichia coli to zinc. Journal of Bacteriology. 2005;187(3):1124-1134. doi:10.1128/JB.187.3.1124.

6. Zhang M, Chen L, Ye C, Yu X. Co-selection of antibiotic resistance via copper shock loading on bacteria from a drinking water bio-filter. Environmental Pollution. 2018;233:132-141. doi:10.1016/j.envpol.2017.09.084.

7. El-Sayed MH. Multiple Heavy Metal and Antibiotic Resistance of Acinetobacter baumannii Strain HAF - 13 Isolated from Industrial Effluents. American Journal of Microbiological Research. 2016;4(1):26-36. doi:10.12691/ajmr-4-1-3.

8. Berg J, Thorsen MK, Holm PE, Jensen J, Nybroe O, Brandt KK. Cu exposure under field conditions coselects for antibiotic resistance as determined by a novel cultivation-independent bacterial community tolerance assay. Environmental Science and Technology. 2010;44(22):8724-8728. doi:10.1021/es101798r. 
9. Zhu YG, Johnson TA, Su JQ, Qiao M, Guo GX, Stedtfeld RD, et al. Diverse and abundant antibiotic resistance genes in Chinese swine farms. Proceedings of the National Academy of Sciences of the United States of America. 2013;110(9):3435-3440. doi:10.1073/pnas.1222743110.

10. Summers AO, Wireman J, Vimy MJ, Lorscheider FL, Marshall B, Levy SB, et al. Mercury released from dental 'silver' fillings provokes an increase in mercury- and antibiotic-resistant bacteria in oral and intestinal floras of primates. Antimicrobial Agents and Chemotherapy. 1993;37(4):825-834. doi:10.1128/AAC.37.4.825.

11. Peltier E, Vincent J, Finn C, Graham DW. Zinc-induced antibiotic resistance in activated sludge bioreactors. Water Research. 2010;44(13):3829-3836. doi:10.1016/j.watres.2010.04.041.

12. Besaury L, Bodilis J, Delgas F, Andrade S, De la Iglesia R, Ouddane B, et al. Abundance and diversity of copper resistance genes cusA and copA in microbial communities in relation to the impact of copper on Chilean marine sediments. Marine Pollution Bulletin. 2013;67(1-2):16-25. doi:10.1016/j.marpolbul.2012.12.007.

13. Seiler C, Berendonk TU. Heavy metal driven co-selection of antibiotic resistance in soil and water bodies impacted by agriculture and aquaculture. Frontiers in Microbiology. 2012;3(DEC):1-10. doi:10.3389/fmicb.2012.00399.

14. Andersson DI, Hughes D. Evolution of antibiotic resistance at non-lethal drug concentrations. Drug Resistance Updates. 2012;15(3):162-172. doi:10.1016/j.drup.2012.03.005.

15. Bengtsson-Palme J, Larsson DGJ. Concentrations of antibiotics predicted to select for resistant bacteria: Proposed limits for environmental regulation. Environment International. 2016;86:140-149. doi:10.1016/j.envint.2015.10.015.

16. Gullberg E, Cao S, Berg OG, Ilbäck C, Sandegren L, Hughes D, et al. Selection of Resistant Bacteria at Very Low Antibiotic Concentrations. PLoS Pathogens. 2011;7(7):e1002158. doi:10.1371/journal.ppat.1002158.

17. Murray AK, Zhang L, Yin X, Zhang T, Buckling A, Snape J, et al. Novel Insights into Selection for Antibiotic Resistance in Complex Microbial Communities. mBio. 2018;9(4):1-12. doi:10.1128/mBio.00969-18.

18. Kraupner N, Ebmeyer S, Bengtsson-Palme J, Fick J, Kristiansson E, Flach CF, et al. Selective concentration for ciprofloxacin resistance in Escherichia coli grown in complex aquatic bacterial biofilms. Environment International. 2018;116(March):255-268. doi:10.1016/j.envint.2018.04.029.

19. Pal C, Bengtsson-Palme J, Kristiansson E, Larsson DGJ. Co-occurrence of resistance genes to antibiotics, biocides and metals reveals novel insights into their co-selection potential. BMC Genomics. 2015;16(1). doi:10.1186/s12864-015-2153-5.

20. Yu Z, Gunn L, Wall P, Fanning S. Antimicrobial resistance and its association with tolerance to heavy metals in agriculture production. Food Microbiology. 2017;64:23-32. doi:10.1016/j.fm.2016.12.009.

21. Summers A. Generally Overlooked Fundamentals of Bacterial Genetics and Ecology. Clinical Infectious Diseases. 2002;34(s3):S85-S92. doi:10.1086/340245. 
22. Stepanauskas R, Glenn TC, Jagoe CH, Tuckfield RC, Lindell AH, King CJ, et al. Coselection for microbial resistance to metals and antibiotics in freshwater microcosms. Environmental Microbiology. 2006;8(9):1510-1514. doi:10.1111/j.1462-2920.2006.01091.x.

23. Pal C, Asiani K, Arya S, Rensing C, Stekel DJ, Larsson DGJ, et al. Metal Resistance and Its Association With Antibiotic Resistance. 1st ed. April. Elsevier Ltd.; 2017. Available from: http://www.sciencedirect.com/science/article/pii/S0065291117300085.

24. FAO and WHO. Joint FAO/WHO Expert Meeting in collaboration with OIE on Foodborne Antimicrobial Resistance: Role of the Environment, Crops and Biocides; 2019.

25. Gerrish PJ, García-Lerma JG. Mutation rate and the efficacy of antimicrobial drug treatment. The Lancet Infectious Diseases. 2003;3(1):28-32. doi:10.1016/S1473-3099(03)00485-7.

26. Bell BG, Schellevis F, Stobberingh E, Goossens H, Pringle M. A systematic review and meta-analysis of the effects of antibiotic consumption on antibiotic resistance. BMC Infectious Diseases. 2014;14(1):13. doi:10.1186/1471-2334-14-13.

27. Ayscue P, Lanzas C, Ivanek R, Grohn YT. Modeling on-farm Escherichia coli O157:H7 population dynamics. Foodborne Pathogens \& Disease. 2009;6(4):461-470. doi:10.1089/fpd.2008.0235.

28. Baker M, Hobman JL, Dodd CER, Ramsden SJ, Stekel DJ. Mathematical modelling of antimicrobial resistance in agricultural waste highlights importance of gene transfer rate. FEMS Microbiology Ecology. 2016;92(4):1-10. doi:10.1093/femsec/fiw040.

29. Murphy JT, Walshe R, Devocelle M. A computational model of antibiotic-resistance mechanisms in Methicillin-Resistant Staphylococcus aureus (MRSA). Journal of Theoretical Biology. 2008;254(2):284-293. doi:10.1016/j.jtbi.2008.05.037.

30. Hellweger F. Simple model of tetracycline antibiotic resistance in aquatic environment: Accounting for metal coselection. Journal of Environmental Engineering. 2013;139(6):913-921.

31. Picioreanu C, Kreft JU, Van Loosdrecht MCM. Particle-based multidimensional multispecies biofilm model. Applied and Environmental Microbiology. 2004;70(5):3024-3040. doi:10.1128/AEM.70.5.3024-3040.2004.

32. Ivask A, Rõlova T, Kahru A. A suite of recombinant luminescent bacterial strains for the quantification of bioavailable heavy metals and toxicity testing. BMC Biotechnology. 2009;9(1):41. doi:10.1186/1472-6750-9-41.

33. R Core Team. R: A Language and Environment for Statistical Computing; 2016. Available from: https://www.R-project.org/.

34. Soetaert K, Petzoldt T, Setzer RW. Solving Differential Equations in R: Package deSolve. Journal of Statistical Software. 2010;33(9):1-25. doi:10.18637/jss.v033.i09.

35. Soetaert K, Herman PMJ. A Practical Guide to Ecological Modelling. Using R as a Simulation Platform. Springer; 2009. 
36. Soetaert K. rootSolve: Nonlinear root finding, equilibrium and steady-state analysis of ordinary differential equations; 2009.

37. Analytics R, Weston S. doParallel: Foreach Parallel Adaptor for the 'parallel' Package; 2015. Available from: https://CRAN.R-project.org/package=doParallel

38. Analytics R, Weston S. foreach: Provides Foreach Looping Construct for R; 2015. Available from: https://CRAN.R-project.org/package=foreach.

39. Hoops S, Sahle S, Gauges R, Lee C, Pahle J, Simus N, et al. COPASI-a COmplex PAthway SImulator. Bioinformatics. 2006;22(24):3067-3074. doi:10.1093/bioinformatics/btl485.

40. Wickham H. ggplot2: Elegant Graphics for Data Analysis. Springer-Verlag New York; 2009. Available from: http://ggplot2.org.

41. Curds CRR. Computer simulations of microbial population dynamics in the activated-sludge process. Water Research. 1971;5(11):1049-1066. doi:10.1016/0043-1354(71)90038-8.

42. Godwin D, Slater JH. The influence of the growth environment on the stability of a drug resistance plasmid in Escherichia coli K12. Journal of general microbiology. 1979;111(1):201-210. doi:10.1099/00221287-111-1-201.

43. Levin BR, Stewart FM, Rice VA. The kinetics of conjugative plasmid transmission: Fit of a simple mass action model. Plasmid. 1979;2(2):247-260. doi:10.1016/0147-619X(79)90043-X.

44. Ibrahim D. Phenotypic and genotypic study of multidrug resistant, extended spectrum $\beta$-lactamase (ESBL)-producing Escherichia coli isolated from a dairy farm; 2017. Available from: http://eprints.nottingham.ac.uk/46613/.

45. Kudva IT, Blanch K, Hovde CJ. Analysis of Escherichia coli O157:H7 survival in ovine or bovine manure and manure slurry. Applied and Environmental Microbiology. 1998;64(9):3166-3174.

46. McDermott PJ, Gowland PC, Gowland PC. Adaptation of Escherichia coli growth rates to the presence of pBR322. Lett Appl Microbiol. 1993;17(3):139-143. doi:10.1016/0305-0491(83)90230-4.

47. Subbiah M, Top EM, Shah DH, Call DR. Selection pressure required for long-term persistence of blaCMY-2-positive IncA/C plasmids. Applied and Environmental Microbiology. 2011;77(13):4486-4493. doi:10.1128/AEM.02788-10.

48. Volkova VV, Lanzas C, Lu Z, Gröhn YT. Mathematical Model of Plasmid-Mediated Resistance to Ceftiofur in Commensal Enteric Escherichia coli of Cattle. PLoS ONE. 2012;7(5):e36738. doi:10.1371/journal.pone.0036738.

49. Nordström K, Molin S, Aagaard-Hansen H. Partitioning of plasmid R1 in Escherichia coli,. Plasmid. 1980;4(2):215-227. doi:10.1016/0147-619X(80)90011-6.

50. Knapp CW, McCluskey SM, Singh BK, Campbell CD, Hudson G, Graham DW. Antibiotic Resistance Gene Abundances Correlate with Metal and Geochemical Conditions in Archived Scottish Soils. PLOS ONE. 2011;6(11):1-6. doi:10.1371/journal.pone.0027300. 
51. Knapp CW, Callan AC, Aitken B, Shearn R, Koenders A, Hinwood A. Relationship between antibiotic resistance genes and metals in residential soil samples from Western Australia. Environmental Science and Pollution Research. 2017;24(3):2484-2494. doi:10.1007/s11356-016-7997-y.

52. Zhao Y, Cocerva T, Cox S, Tardif S, Su JQ, Zhu YG, et al. Evidence for co-selection of antibiotic resistance genes and mobile genetic elements in metal polluted urban soils. Science of The Total Environment. 2019;656:512-520. doi:10.1016/j.scitotenv.2018.11.372.

53. Hooton SP, Millard AD, Baker M, Stekel DJ, Hobman JL. DNA Traffic in the Environment and Antimicrobial Resistance. In: DNA Traffic in the Environment. Springer; 2019. p. 245-271.

54. ALS Environment. Heavy Metal Guidelines in Soil TECHNICAL DATASHEET Assessment of Potentially Toxic Elements; 2019. Available from: www.alsenvironmental.co.uk.

55. Health P, Bilthoven BA. NATIONAL INSTITUTE OF PUBLIC HEALTH AND THE ENVIRONMENT Addendum to RIVM report number 601501 001; 1997. 601501.

56. Nies DH. Microbial heavy-metal resistance. Applied Microbiology and Biotechnology. 1999;51(6):730-750. doi:10.1007/s002530051457.

57. Arya S, Todman H, Baker M, Hooton S, Millard A, Kreft JU, Hobman JL, Stekel DJ. A generalised model for generalised transduction: the importance of co-evolution and stochasticity in phage mediated antimicrobial resistance transfer. FEMS Microbiology Ecology. 2020;fiaa100. doi:10.1093/femsec/fiaa100.

58. Ye J, Rensing C, Su J, Zhu YG. From chemical mixtures to antibiotic resistance. Journal of Environmental Sciences (China). 2017;62:138-144. doi:10.1016/j.jes.2017.09.003.

59. Williams O, Clark I, Gomes RL, Perehinec T, Hobman JL, Stekel DJ, et al. Removal of copper from cattle footbath wastewater with layered double hydroxide adsorbents as a route to antimicrobial resistance mitigation on dairy farms. Science of the Total Environment. 2019;655:1139-1149. 